\title{
Un ambassadeur de France en Italie : Gaston Palewski (août 1957-avril 1962)
}

Un ambasciatore francese in Italia: Gaston Palewski (agosto 1957-aprile 1962)

A French Ambassador in Italy: Gaston Palewski (August 1957-April 1962)

\section{Élisabeth Yverneau-Glasser}

\section{OpenEdition Journals}

Édition électronique

URL : http://journals.openedition.org/cei/2814

DOI : $10.4000 /$ cei.2814

ISSN : 2260-779X

Éditeur

UGA Éditions/Université Grenoble Alpes

Édition imprimée

Date de publication : 20 avril 2016

Pagination : 47-60

ISBN : 978-2-84310-323-0

ISSN : $1770-9571$

Référence électronique

Élisabeth Yverneau-Glasser, « Un ambassadeur de France en Italie : Gaston Palewski (août 1957-

avril 1962) », Cahiers d'études italiennes [En ligne], 22 | 2016, mis en ligne le 01 janvier 2017, consulté le 26 mars 2021. URL : http://journals.openedition.org/cei/2814 ; DOI : https://doi.org/10.4000/cei.2814 


\title{
UN AMBASSADEUR DE FRANCE EN ITALIE : GASTON PALEWSKI (AOÛT 1957-AVRIL 1962)
}

\author{
Elisabeth Yverneau-Glasser \\ Professeur agrégé, docteur en histoire
}

"Je revis les cinq années au cours desquelles j'ai eu l'honneur de représenter la France en Italie. Elles resteront pour moi, un souvenir inoubliable. J'ai été heureux en particulier que la croissance prodigieuse de l'économie italienne coïncide avec le renfort des échanges entre nos deux pays [...]. À la fin de la haute mission qui m'a été confiée, je demeure convaincu que la nécessité de coopération entre nos voisins constitue une des données les plus importantes de notre politique et notamment de notre politique européenne.»

Gaston PALEwski, discours à la foire de Paris, 23 mai 1962, AN, 547 AP 69.

"L'ambassade de Gaston Palewski à Rome, une longue mission selon les normes actuelles [...] demeurera dans l'histoire comme un épisode heureux des relations franco-italiennes [...]. Ce grand serviteur de la France était aussi un grand ami de l'Italie.»

Étienne Burin des Roziers, "Ambassadeur à Rome», Espoir, nº 50, I985, p. 3I.

Après avoir été dans l'entourage de deux grands hommes, le maréchal Lyautey (en I924-I925) puis Paul Reynaud (entre I928 et 1940), Gaston Palewski sert la France aux côtés du général de Gaulle. Il rejoint celui-ci à Londres dès 1940 et devient son directeur de cabinet de septembre 1942 à janvier 1946. Fondateur, militant et député du RPF entre 1947 et 1955, il occupe de février à octobre 1955 le poste de ministre d'État chargé de la 
Recherche et des Questions atomiques et spatiales dans le gouvernement d'Edgar Faure. En août 1957, Gaston Palewski est nommé par le ministre des Affaires étrangères, Christian Pineau, "ambassadeur extraordinaire et plénipotentiaire de la République française en Italie pour une durée de six mois, renouvelable ${ }^{\mathrm{I}}$. C'est pour lui une nouvelle responsabilité, celle de représenter la France sur la scène internationale.

Gaston Palewski succède à Jacques Fouques-Duparc qui occupait cette charge depuis une dizaine d'années. Il s'installe dans la capitale italienne le 15 octobre 1957 et réside au palais Farnèse, un des plus beaux bâtiments de la ville aux conditions d'accueil exceptionnelles. C'est une ambassade de prestige accordée généralement en récompense à des hommes fidèles au gouvernement. À Rome, les trois ambassadeurs qui se succèdent entre I957 et 1969 ont servi, chacun à un titre ou à un autre, le général de Gaulle. C'est le cas de Gaston Palewski de 1957 à 1962, d'Armand Bérard de 1962 à 1967, puis à partir de 1967 d'Étienne Burin des Roziers. Avec un séjour de presque cinq ans, Gaston Palewski a effectué un des plus longs mandats d'ambassadeur de France à Rome, celui de son prédécesseur constituant une exception.

Nommé quelques mois avant le retour au pouvoir du général de Gaulle par le président du Conseil Bourgès-Maunoury, compagnon de la Libération et ancien ministre de l'Intérieur du second gouvernement Edgar Faure, Gaston Palewski accomplit sa mission dans le contexte de la guerre d'Algérie et des débuts de la construction européenne. C'est à Rome qu'il assiste en "spectateur» au retour au pouvoir du général de Gaulle et à la naissance de la Cinquième République en France.

Qui est ce nouvel ambassadeur et quelle est sa mission? Comment Gaston Palewski, gaulliste de la première heure, militant, ministre, amateur d'art, homme de réseau est-il accueilli? Quelle est la nature des relations franco-italiennes? Quelle est la place de l'Italie sur le plan international et dans le cadre européen?

Dans ses Mémoires ${ }^{2}$ Gaston Palewski consacre seulement dix pages à cette période et évoque surtout les personnalités italiennes qu’il a rencontrées. Ses archives privées contiennent nombre de discours et maintes invitations. Nous avons complété ces sources par les archives diplomatiques conservées au ministère des Affaires étrangères, série Z, EuropeItalie (1944-1947) et par les Documents diplomatiques français.

I. Décret du I7 août 1957, publié au Journal officiel du I8 août 1957.

2. G. Palewski, Mémoires d'action, 1924-1974, Paris, Plon, 1988. 


\section{Qui est ce nouvel ambassadeur? Quelle est sa mission?}

Lorsque Gaston Palewski est nommé ambassadeur, il sort d'une période d'inaction politique. Il a démissionné de ses fonctions de ministre d'État chargé de la Recherche et des Questions atomiques et spatiales en octobre I955. En janvier 1956, il n’est pas réélu député. Il n’exerce aucune fonction politique ou administrative, il entretient ses réseaux personnels et poursuit probablement une partie de ses activités d'influence.

Gaston Palewski connaît peu l'Italie, État fondateur avec la France de la Communauté économique européenne et membre de l'Alliance atlantique. Les rapports entre les deux nations latines n'ont pas toujours été simples. Après la Seconde Guerre mondiale, l'Italie mène une politique atlantiste, ses dirigeants privilégiant les relations transatlantiques et recherchant une influence plus étendue en Méditerranée. Dans ses Mémoires, Gaston Palewski évoque un court séjour avant la guerre à l'occasion d'une visite à un ami et précise qu'il a conservé "un délicieux souvenir ${ }^{3}$ " de ce pays. Il a aussi accompagné, début août 1944 le général de Gaulle à Naples. Avoir eu, au préalable, une expérience italienne ne semble pas avoir été, pour les ambassadeurs qui se sont succédé au palais Farnèse après la Seconde Guerre mondiale, un élément déterminant de leur affectation (excepté Gilles Martinet dans les années 1980, gendre de Bruno Buozzi).

Gaston Palewski a derrière lui presque trente années d'expérience politique, il a vu fonctionner les assemblées législatives de la Troisième et de la Quatrième République. Il connait également des milieux politiques à l'étranger (Afrique du Nord, Royaume-Uni, URSS, États-Unis). Même si sa formation ne le prédestinait pas à une carrière diplomatique, ses expériences antérieures en France et à l'étranger, son réseau de relations personnelles sont autant d'atouts pour sa nomination au palais Farnèse, dans un contexte tendu, celui de la décolonisation et notamment de la guerre d'Algérie. Au-delà des enjeux politiques, sa culture, ses connaissances historiques, ses qualités relationnelles ont certainement pesé dans sa nomination dans une ambassade d'un pays européen chargé d'histoire.

Gaston Palewski doit cette nomination au général de Gaulle, pourtant retiré à Colombey-les-Deux-Églises. Étienne Burin des Roziers précise que Gaston Palewski «n'aurait rien fait sans l'accord du général de Gaulle, rêvait d'être ambassadeur ${ }^{4}$. Gaston Palewski écrit, début août 1957, à son

3. Ibid., p. 268.

4. Entretien avec Étienne Burin des Roziers, Paris, 6 octobre 2005. 
cousin Maurice André-Gillois : «Je suis heureux de devoir cette belle occasion d'agir et, je l'espère de servir, à l'action généreuse, une fois de plus, du général de Gaulles."

Gaston Palewski est satisfait de cette nomination qui lui permet de revenir dans l'appareil de l'État, de retrouver une fonction officielle, prestigieuse et d'être au service de son pays même si c'est en dehors de l'Hexagone. Cette nouvelle fonction est une étape supplémentaire dans son itinéraire personnel qui révèle au fil des années une carrière ascendante. Tout en manifestant sa satisfaction, il exprime aussi quelques regrets, en particulier celui de quitter la France, et surtout Paris.

$\mathrm{Au}$ moment où Gaston Palewski prend ses fonctions, les questions frontalières sont réglées et les deux pays, membres fondateurs de la Communauté économique européenne, se sont rapprochés. Depuis décembre 1947, l'Italie a une nouvelle Constitution qui fait du pays une République démocratique et parlementaire dans laquelle les pouvoirs du président de la République sont réduits. Aucune majorité homogène ne se dégage et Gaston Palewski décrit la situation en affirmant : «Les partis régnaient, tout-puissants, sur un exécutif faible et incertain» et dénonce une Italie "dépourvue d'exécutif ${ }^{6}$ ".

L'Italie réagit plutôt favorablement à sa nomination si on analyse la lettre de Giuseppe Pella, ministre italien des Affaires étrangères : «La désignation d'une personnalité aussi connue que Monsieur Palewski, d'une valeur et d'une capacité très grandes dans le domaine politique, est apparue de prime abord comme une confirmation éclatante de l'importance attribuée au poste de Rome par le gouvernement de Paris 7 ."

Gaston Palewski a un jugement plus nuancé sur les réactions suscitées et évoque : "L'inquiétude des Italiens qui voyaient en moi un adversaire des communautés européennes ${ }^{8}$.» Étienne Burin des Roziers confirme cette impression. Le fait qu'il ne soit pas «de la carrière», mais un gaulliste historique, vaut à Gaston Palewski d'être accueilli fraîchement au palais Farnèse. Conscient de succéder à un ambassadeur qui a marqué l'Italie et de ne pas être issu du corps diplomatique, il réunit la colonie française de Rome «un peu effarouchée par l'arrivée d'un homme politique à la place des tenants habituels de la carrière».

5. AN, 547 AP 64.

6. G. Palewski, Mémoires, ouvr. cité, p. 270.

7. Archives du Quai d'Orsay, série Europe, sous-série Italie, I956-I960, dossier 274, lettre du 27 juillet I957, signée Pella.

8. G. Palewski, Mémoires, ouvr. cité, p. 269. 
Le I3 octobre 1957, Louis Joxe, secrétaire général du Quai d'Orsay envoie au nouvel ambassadeur les instructions du ministre indiquant les "principales lignes d'orientation» de sa mission. Il lui précise :

Le pays dans lequel vous avez la charge de représenter la France est uni à cette dernière, non seulement par son passé, par tous les liens géographiques, ethniques et historiques qui résultent d'un voisinage immédiat, mais aussi par son avenir, par une communauté de destin qui n'a, sans doute, jamais été plus étroite qu'aujourd'hui ${ }^{9}$.

Les liens historiques sont anciens et Louis Joxe souligne l'existence d'une coopération qui doit aller «en se développant dans le cadre de l'Europe et de l'Eurafrique». Les relations entre la France et l'Italie doivent s'inscrire dans un cadre bilatéral, mais aussi avoir une dimension internationale et en particulier européenne. Louis Joxe indique aussi que «tout ce qui touche à notre action en Algérie devra retenir votre attention par priorité». Pour Gaston Palewski, habitué à des relations bilatérales et privilégiant la relation personnelle, c'est un mode nouveau de fonctionnement. Mais c'est aussi quelque chose d'exaltant, car il va pouvoir agir, intervenir, se déplacer. La situation de l'Algérie occupe effectivement une place importante dans son activité d'ambassadeur, dont il doit informer très régulièrement le Quai d'Orsay.

\section{Un ambassadeur au début de la Cinquième République}

C'est à Rome, où il est ambassadeur depuis huit mois, que Gaston Palewski apprend le retour au pouvoir du général de Gaulle, retour qui se situe au paroxysme de la crise en Algérie. Ce retour provoque une certaine appréhension en Italie. Il est interprété, surtout par la gauche italienne, comme une atteinte à la démocratie. Il y a une crainte de voir le Général diriger personnellement la France en concentrant les pouvoirs entre ses mains et les Italiens redoutent l'apparition d'une sorte de fascisme. Cette inquiétude est confirmée par l'ambassadeur italien à Paris, Giovanni Fornari : "L'avènement de Charles de Gaulle suscitait quelques perplexités en Italie. On redoutait de voir surgir en France une forme de fascisme ${ }^{\mathrm{IO}}$."

Dans une lettre manuscrite adressée au général de Gaulle, Palewski écrit : "Mon Général, c'est un crève-cœur pour moi, après tant d'années où j'ai appelé de tout mon être ces heures décisives, d'être loin de vous en

9. Archives du Quai d'Orsay, série Europe, sous-série Italie, I956-1960, dossier 274, instruction donnée au nouvel ambassadeur de France à Rome, I3 octobre 1957.

Io. G. Fornari, «Le dernier des Stendhaliens", Espoir (Revue de l'Institut Charles de Gaulle), nº 50, I985, p. 52. 
ce moment. Dès qu'il me sera possible de venir, j'accourrai vers vous. En attendant, je m'efforce ici de travailler ${ }^{\mathrm{II}}$.» On sent le regret de ne pas être en France en mai-juin 1958.

Ce retour du général de Gaulle est l'occasion pour Gaston Palewski de prononcer un long discours le I4 juillet 1958 devant la colonie française de Rome ${ }^{12}$. C'est son premier discours en tant qu'ambassadeur le jour de la fête nationale. Il l'organise autour de cinq points : la maladie de l'État, de Gaulle garant des libertés publiques, la Constitution et le problème algérien, les relations de la métropole avec le continent africain, la coopération franco-italienne. Les années 1957-1958 ne sont pas faciles pour lui et il éprouve quelques difficultés à trouver une certaine crédibilité dans ses fonctions au moment où la France change de Constitution.

Lorsque Gaston Palewski arrive au palais Farnèse, la guerre d'Algérie dure depuis trois ans. C'est un dossier délicat que l'ambassadeur doit gérer. Les instructions du Quai d'Orsay, d'octobre 1957, montrent l'importance primordiale de cette question dans les rapports avec l'Italie. Nous pouvons y lire : "Tout ce qui touche à notre action en Algérie devra retenir votre attention par priorité. Vous aurez soin de me communiquer toutes informations qui pourraient nous être utiles dans ce domaine ${ }^{\mathrm{r}}$." Il est demandé à l'ambassadeur de recueillir tous les renseignements sur la situation en Algérie et cela constitue une part de ses activités jusqu'en 1962. En 1958, la priorité du Général est de mettre fin à la guerre d'Algérie qui est un «boulet» et un «handicap ${ }^{14}$ ». L'Italie est préoccupée par la tournure des événements algériens, car elle considère la Méditerranée comme son domaine naturel d'influence.

L'ambassadeur transmet à Paris de nombreux rapports sur les réactions de l'opinion publique italienne face aux événements algériens. Le jugement des Italiens a fluctué au gré des mesures adoptées par les autorités françaises et nous pouvons noter, à travers cette correspondance, plusieurs étapes. Entre le déclenchement de la guerre d'Algérie et le milieu de l'année 1956, donc avant son arrivée, on constate une certaine indifférence des Italiens dans la Péninsule face aux problèmes rencontrés par la France en Algérie. À partir du milieu de l'année 1956, l'Italie reconnaît toutefois la légitimité des revendications des nationalistes algériens et en 1957, année de l'arrivée de Gaston Palewski à Rome, il y a un véritable retournement

II. AN, $547 \mathrm{AP} 6$ I. Le document n'est pas daté.

I2. Archives du Quai d'Orsay, série Europe, sous-série Italie, 1956-1960, relations franco-italiennes, volume 30I.

I3. Ibid., volume 274, corps diplomatique, instruction générale donnée à Gaston Palewski, 29 octobre 1957.

I4. M. Vaïsse, La grandeur. Politique étrangère du général de Gaulle, I958-1969, Paris, Fayard, I998, p. 60 et 63. 
de situation : l'opinion italienne bascule du côté des nationalistes algériens en lutte et multiplie les griefs contre la France ${ }^{15}$. Mais l'Italie ne remet jamais en cause l'amitié entre les deux pays. L'ambassadeur fait remonter à Paris toutes les récriminations de la presse : «La France est incapable de régler le problème», "La France s'enlise dans une guerre sans espoir», "La France utilise la torture ${ }^{16}$. En arrivant à Rome, il fait tous ses efforts pour persuader l'Italie de soutenir la politique française en Afrique du Nord. Il reçoit régulièrement à l'ambassade les journalistes et intellectuels influents qu'il essaie de convaincre. La crise de mai 1958 en Algérie et l'appel au général de Gaulle provoquent une "émotion considérable» dans la presse italienne qui donne une vision dramatique de la situation. L'ambassadeur s'exprime sur le problème algérien dans le cadre de conférences organisées à travers la Péninsule. Dans ses Mémoires, il cite Milan, Florence, Turin, Gênes, Venise.

En 1958, la politique française en Algérie est très critiquée par l'Italie, terre d'accueil et base arrière pour le FLN, ainsi que carrefour pour le trafic d'armes à destination de l'Algérie. Certains milieux italiens sont particulièrement disposés à offrir leur hospitalité aux représentants du FLN qui ont leurs bureaux à Rome sous le couvert de l'ambassade de Tunisie. Le Palais Farnèse intervient auprès des autorités italiennes pour protester. En octobre 1958 se pose le problème du transit de Fehrat Abbas, président du GPRA. Gaston Palewski demande à être reçu par le président du Conseil italien Amintore Fanfani pour que Fehrat Abbas ne puisse quitter l'aéroport de Ciampino ${ }^{17}$. Non sans difficultés, l'ambassadeur obtient que le gouvernement italien notifie à Fehrat Abbas qu'il ne pourra sortir de l'aéroport. Cependant, l'ambassade n'a pas toujours obtenu satisfaction sur la question du transit des personnalités indépendantistes algériennes.

La pression des Italiens se relâche quand les négociations s'ouvrent entre la France et le FLN. Au cours de l'année 1960, les Italiens sont soulagés de voir le général de Gaulle évoluer vers une solution négociée, mais certains journaux craignent qu'il ne puisse surmonter les oppositions et l’optimisme reste modéré. Le référendum de janvier i96I sur l'autodétermination est approuvé en Italie. L'ambassade note : «L'opinion italienne a accueilli avec un sentiment de soulagement les résultats du referendum.

I5. Voir M. Vaïsse, Le poids de la guerre d'Algérie dans la politique extérieure de la France (1958-1962), Institut Charles de Gaulle (éd.), De Gaulle en son siècle, vol. VI, Paris, Plon, 1992.

I6. Archives du Quai d'Orsay, série Europe, sous-série Italie, volume 306, dépêche 506, Rome, 28 mars 1958.

17. Documents diplomatiques français, 1958, tome 2, pages 399-400. 
L'Italie est parmi les alliés de la France l'un des pays les plus intéressés à une solution rapide du problème algérien ${ }^{18}$.»

Les résultats du référendum de 1962 concernant les accords d'Évian sont accueillis favorablement par l'opinion italienne et le prestige du Général est renforcé.

L'ambassade de France à Rome a suivi de très près les événements d'Algérie en faisant remonter toutes les informations vers Paris. C'est quelques semaines après la signature des accords d'Évian que Gaston Palewski est rappelé à Paris. Pour lui être à Rome relève au départ d'un concours de circonstances, puis cela a permis au général de Gaulle d'avoir un homme sûr afin de suivre le déroulement des événements algériens, d'avoir en Italie quelqu'un qui connaît l'Afrique du Nord.

Pour commémorer le centenaire des batailles de Magenta et de Solferino ${ }^{19}$ le général de Gaulle effectue, du 23 au 26 juin 1959, un déplacement en Italie : "Voyage historique» selon Giovanni Fornari, «voyage qui marque un tournant dans l'histoire des relations franco-italiennes" selon Étienne Burin des Roziers ${ }^{20}$. Ce séjour est le premier voyage officiel du Général en tant que président de la Cinquième République. En ce sens, il revêt une importance symbolique toute particulière et montre l'intérêt que la France porte aux relations avec l'Italie. Le Général est accompagné de Maurice Couve de Murville et de Pierre Guillaumat. Le 24 juin I959, le Général confie au président italien Giovanni Gronchi que le but de ce voyage "est de voir comment les deux pays peuvent coopérer ${ }^{2 \mathrm{I}}$. Il y a donc un objectif politique à ce déplacement, celui d'étudier la coopération, la collaboration économique et politique entre les deux États.

Un ambassadeur est traditionnellement attaché aux visites officielles des plus hautes personnalités de son pays, et Gaston Palewski n'a pas fait exception à la règle. C'est lui qui prépare et met en œuvre cette visite placée sous le signe de la coopération franco-italienne. Le voyage était prévu avant le retour au pouvoir du General puisqu'il avait été envisagé dès août 1957. Gaston Palewski avait évoqué le projet de commémoration peu de temps après son arrivée et voulait donner à ces manifestations un certain éclat, car les deux batailles avaient, avec le concours de la France,

I8. Archives du Quai d'Orsay, série Europe, sous-série Italie, I96I-I970, volume 359, document du I2 janvier I96I.

I9. Bataille de Magenta : victoire des Français de Napoléon III sur les Autrichiens, en Lombardie, 4 juin I859. Bataille de Solferino : victoire française sur les Autrichiens, en Lombardie, 24 juin I859.

20. É. Burin des Roziers, "Ambassadeur à Rome", Espoir, nº 50, 1985, p. 32.

2I. Documents diplomatiques français, vol. I, $\mathrm{n}^{\circ} 37 \mathrm{I}$, 1959 . 
ouvert la voie de l'unité et de l'indépendance italienne. Il a suivi très régulièrement l'organisation de cet événement.

Au cours de son déplacement le Général rend hommage aux Français et aux Italiens, et dans les discours qu'il prononce à Milan, Solferino et Rome, il manifeste son attachement aux valeurs de liberté et de démocratie. Il insiste sur l'ancienneté du rapprochement franco-italien et les communautés de vues entre les deux États. Il exalte l'Italie moderne, dynamique et insiste sur la nécessité de développer une assistance économique et technique entre les deux pays et entre les États signataires du traité de Rome.

Alors que Gaston Palewski pensait que «l'Italie était, sans doute, parmi les pays de l'Europe occidentale, l'un de ceux où l'on pouvait craindre que le président de la République reçût l'accueil le plus réservé22 ", le voyage est triomphal et enthousiaste. Les foules sont nombreuses. La presse italienne, excepté celle du parti communiste, consacre une place considérable à l'événement qu'elle présente sous un angle très positif et emblématique de la coopération franco-italienne. L'ambassadeur souligne les «longs commentaires" positifs à propos du chef de l'État, "les abondantes biographies du Général» et relève qu’à Rome, depuis longtemps, aucune visite officielle n'a retenu à ce point l'attention populaire.

Le Général écrit à Gaston Palewski le I2 juillet 1959 pour le féliciter de l'organisation du voyage : "Je veux dire combien j'ai été satisfait de la façon dont vous avez préparé, déclenché et mis en œuvre mon voyage en Italie [...]. Très manifestement vous avez, en personne, une position tout à fait exceptionnelle chez eux ${ }^{23}$.»

Les questions européennes constituent la toile de fond de l'ambassade de Gaston Palewski dans un pays fondateur de la CEE. Il communique très régulièrement au Quai d'Orsay les remarques, les interrogations des Italiens sur la mise en place du traité de Rome. Il favorise une politique économique bilatérale consistant à promouvoir les exportations et à soutenir les entreprises françaises qui souhaitent s'engager sur le marché italien. En mai 1959, il informe que l'Italie est favorable au renforcement du Marché commun mais qu'elle émet quelques réserves sur les modalités de l'accélération du processus, car elle craint une accentuation des contrastes entre le nord et le sud de la Péninsule. Il précise aussi que le pays souhaite sauvegarder ses intérêts dans le commerce avec les pays européens étrangers au Marché commun. Il note des résultats moins encourageants

22. Archives du Quai d'Orsay, série Europe, sous-série Italie, I944-I970, volume 300, lettre du 2 juillet 1959.

23. Ch. de Gaulle, Lettres, notes et carnets, juin 1958-décembre 1960, Paris, Plon, 1985, p. 238. 
à propos de l'agriculture italienne et en août I960 il informe le ministère que l'Italie n'est pas prête pour la Politique agricole commune.

\section{Une diplomatie de prestige}

À Rome, Gaston Palewski déploie une «diplomatie de prestige». Il a une réputation de mondain, il veut séduire, impressionner. Il reçoit beaucoup et effectue de très nombreux déplacements : "Je compris tout de suite que je ne serais égal à mes fonctions qu'allant de ville en ville pour manifester à tous et à chacun l'amitié de la France ${ }^{24}$."

Gaston Palewski a conservé de nombreuses traces de ses voyages et des discours qu'il a prononcés. Il est à l'aise dans sa charge, satisfait de son poste d'ambassadeur. C'est cet aspect qui ressort de la lecture de ses Mémoires et du dépouillement des archives. Il inaugure de nombreuses usines (une usine de transistors à Sermoneta en octobre 1959, Saint-Gobain à Caserte en décembre 1959, les établissements L'Oréal à Turin en mai 1960), des expositions (Édouard Vuillard à Milan en septembre 1959, une exposition d'artisanat à Florence en avril I960, l'exposition Daumier à Milan en mai et juillet 196I). Certaines inaugurations concernent le domaine de la culture : le Centre culturel franco-italien en octobre 1959, les locaux de l'Institut français de Florence en janvier 196I, l'Association francoitalienne de Bologne et l'École française de Milan en octobre I96ı. Il est présent lors de nombreuses foires-expositions : la journée française de la foire de Bari en septembre 1958, celle de Milan en avril 1959, la journée française de la foire agricole de Venise en mars 1959, les foires de Vérone en mars 1960, de Milan en avril 1960, de Bari en août 1960, de Palerme en mai I96I. Il signe la convention franco-italienne concernant l'aménagement du Mont-Cenis en juillet 1960 et visite les chantiers du tunnel sous le Mont-Blanc en décembre 1960. Les nombreux déplacements qu’il accomplit concernent tous les domaines, politique, économique, social, culturel. Gaston Palewski a une bonne connaissance de l'Italie, de son histoire, de sa culture, des villes qu'il visite. Il fait régulièrement une référence artistique et culturelle de la ville dans laquelle il se rend lorsqu'il prononce un discours. Il dispose toujours d'une note pour préparer son voyage, sur les richesses culturelles et artistiques de telle ou telle cité, des renseignements sur un peintre, un écrivain, un artiste.

24. AN, 547 AP 57, AP 58 et AP 59. 
Gaston Palewski a beaucoup sillonné l'Italie et n’a jamais négligé les visites de musées, d'églises, d'expositions, de sites archéologiques. Il s'est entretenu avec les représentants des pouvoirs publics, protagonistes de la vie locale, hommes d'affaires, syndicalistes.

Gaston Palewski reçoit beaucoup à l'ambassade et avec un certain éclat. Étienne Burin des Roziers confirme qu'il «recevait très bien et il aimait recevoir ${ }^{25}$ ». D'après Giovanni Fornari : «Le Farnèse fut pour lui une résidence idéale où put se donner libre cours l'art exquis qu'il possédait de maintenir et d'embellir les intérieurs, d'en faire les honneurs à ses innombrables amis, de leur présenter les nouvelles pièces qu'il ne cessait de dénicher au cours de ses randonnées à travers Rome ${ }^{26}$." Gaston Palewski affirme que "c'est la plus belle ambassade de France», un «merveilleux palais ${ }^{27}$ " et qu'il lui a été "agréable de le rendre vivant, de le réchauffer, d'y mettre ses tableaux, d'y grouper ceux qui auraient pu s'y trouver dans d'autres époques ${ }^{28}$ ".

Gaston Palewski s'est beaucoup intéressé à l'histoire du palais et il a procédé à la restauration de la galerie des Carrache ${ }^{29}$. Il acquiert, en I959, deux tapisseries d'Aubusson pour le couloir d'accès à cette galerie ${ }^{30}$. Dans une lettre adressée à André Malraux, ministre de la Culture en février I959, il évoque les derniers travaux de réfection et la «remise en état de l'ancienne chapelle du cardinal Farnèse, qui avait été profanée». Dans une lettre du 7 février 1959, adressée à la marquise de Brémond d'Ars, il donne le détail des travaux accomplis :

J'ai éclairé la petite cour intérieure. Je l'ai transformée en jardin à l'italienne avec des lauriers et des ifs [...]. Entre les deux tapisseries d'après le Raphäl des chambres du Vatican, j'ai suspendu deux tableaux de ma collection [...]. L'un d'eux qui représente l'Arc de Titus et les fouilles du forum fut acheté par moi à l'âge de i8 ans, quand j'étais étudiant à l'université d'Oxford : prédestination ${ }^{31}$.

Il précise toutes les transformations qu'il a fait accomplir, et en particulier qu'il a fait repeindre le deuxième étage.

25. Témoignage d'Étienne Burin des Roziers, Paris, 6 octobre 2005 .

26. G. Fornari, «Le derniers des Stendhaliens", art. cité, p. 52.

27. G. Palewski, "Quelques hôtes au palais Farnèse», discours, 7 février 1969, Archives municipales de Nice, I68 W II9.

28. Interview de Gaston Palewski par Jacques Chancel, Radioscopie, I6 juin 1975, Archives de l'INA, Bibliothèque nationale de France.

29. Peintres italiens du Xvi ${ }^{\mathrm{e}}$ siècle originaires de Bologne. C'est Annibal Carrache (1560-I605) qui décora la galerie du palais Farnèse, en particulier la voûte avec sa version des Amours des dieux, inspirée des Métamorphoses d'Ovide. Plus d'informations à ce sujet sur <www.lemonde.fr/culture/article/2015/05/28/dans-la-galerie-descarrache-a-rome-les-dieux-renaissent_4642687_3246.html>.

30. AN, 547 AP I62.

31. AN, 547 AP 68. 
Gaston Palewski a beaucoup invité au palais Farnèse comme en témoigne l'abondante correspondance ${ }^{32}$ reçue pour le remercier de son accueil ainsi que les menus et plans de table conservés dans ses archives personnelles.

Les Mémoires de son frère Jean-Paul confirment sa "passion" pour cette résidence.

Dès le moment où il fut nommé Ambassadeur à Rome, votre oncle transforma le palais Farnèse et lui rendit tout l'éclat que possédait jadis cette merveilleuse demeure [...]. La maison prit un air de fête et une splendeur depuis longtemps inaccoutumée. Gaston Palewski recevait beaucoup : les réunions de l'Ambassade de France devinrent vite l'un des centres principaux de la vie romaine, il fut un hôte magnifique dont on gardera longtemps le souvenir [...]. Sa discrétion, son goût secret, l'intérêt extrême qu'il manifestait pour toutes les merveilles de l'Antiquité, la Renaissance et le siècle de Tiepolo ont fait jaillir du sol italien, l'intérêt qu'il portait aux réalisations industrielles et à la vie moderne [...]. On le voyait inaugurer des expositions, des foires internationales, s'attarder auprès des temples grecs et des ruines romaines, on le voyait au Nord discuter avec les industriels, au Sud rêver tout à la fois aux douceurs du climat et à la misère du peuple ${ }^{33}$.

Au cours de son séjour en Italie, sa passion pour les lettres et les arts transparaît dans ses engagements, ce que confirme son courrier à Julien Cain, administrateur à la Bibliothèque nationale, en 1968 : «Mon destin a voulu ne jamais séparer les arts de la politique ${ }^{34}$.»

Gaston Palewski s'est aussi beaucoup attaché à la ville de Venise. Elle fut, d'après Giovanni Fornari «sa passion ${ }^{35}$ ». "Venise a joué un rôle particulièrement privilégié dans la vie de Gaston Palewski. Tout l'attirait [...]. Son passé fascinait l'amateur d'histoire. Ses beautés artistiques séduisaient l'homme de goût. La place qu'y tiennent la femme et l'amour enchantait le séducteur ${ }^{36}$.» Ambassadeur, il s'est intéressé à la cité de la lagune menacée par les périls naturels et s'y est rendu de nombreuses fois : en décembre 1958, juillet 1959, février et mars $196 \mathrm{I}^{37}$. Suite aux inondations de 1966, il fonde en 1967, alors qu'il est président du Conseil constitutionnel, le Comité français pour la sauvegarde de Venise.

32. De nombreuses lettres le gratifient personnellement et évoquent "les belles réceptions», "aimable réception au palais Farnèse", "nous avons admiré la splendeur de l'ambassade, le goût que vous avez apporté à son installation et le caractère agréable que vous lui avez donné", "gentillesse de votre accueil", "sympathique accueil», "accueil si sympathique que vous nous avez réservé» (AN, 547 AP 56, AP 66, AP I62).

33. Extrait des Mémoires non publiées de Jean-Paul Palewski, document fourni par Stanislas Palewski.

34. AN, 547 AP I20.

35. G. Fornari, "Le dernier des Stendhaliens », art. cité, p. 53.

36. G. Gaussen, "Au Comité français pour la sauvegarde de Venise», Espoir, nº 50, 1985, p. 50.

37. AN, 547 AP 57 et AP 69. 
Avec Armand Bérard, son successeur de 1962 à 1967, Gaston Palewski pourrait écrire : "L'Italie est, de tous les postes diplomatiques, un des plus délicats. Les oppositions n'y sont pas tranchées, ni les contrastes accusés ${ }^{38}$.» Pour Gaston Palewski, jeune diplomate, fort de ses expériences variées au sein du monde politique français, de sa culture très internationale, la période d'adaptation a sans doute été peu facile. Le monde de la diplomatie ne lui était pas aussi familier qu'il avait pu penser.

Il abandonne ses fonctions diplomatiques quelques semaines après la signature des accords d'Évian. Le général de Gaulle le rappelle à Paris pour le nommer à un ministère clé qu'il a déjà occupé : en avril I962, le nouveau premier ministre Georges Pompidou lui offre le portefeuille de ministre d'État chargé de la Recherche scientifique et des Questions atomiques et spatiales.

On note chez Gaston Palewski une satisfaction certaine de rentrer à Paris, où il retrouve le Général : "Je suis heureux de retrouver Paris ${ }^{39}$." Il a passé cinq années extraordinaires à Rome. Prenant congé de la colonie française de Rome en avril 1962, il exprime néanmoins ses regrets de quitter la Ville éternelle : "Je ne vous cacherai pas ma tristesse. Comment pourrait-on quitter sans mélancolie, après cinq ans, ce palais si noble, cette ville si belle, ce pays si attachant?" Il dresse un bilan plutôt positif de la période malgré le contexte de la tragédie algérienne. Il rappelle la mise en route du Marché commun et note :

Notre rôle est de faire que l'amitié franco-italienne se développe et s'approfondisse au sein de la coopération européenne [...]. Échanges économiques importants et fructueux [...]. Voyage de De Gaulle il y a trois ans [...]. Renouveau de l'étude du français en Italie [...]. Pendant ces cinq années nous avons vu l'Italie se transformer sous nos yeux, passer dans le peloton de tête des nations industrielles d'Europe ${ }^{40}$.

Giovanni Fornari dresse lui aussi, mais avec recul du temps, le bilan vu du côté italien :

En avril 1962, Gaston Palewski achevait donc sa brillante mission en Italie où son amitié pour notre pays avait été vivement appréciée et chaleureusement payée de retour. Les Romains se souviennent qu'à la réception de fin de mission au Farnèse, plus de deux mille personnes avaient envahi les admirables salons pour exprimer à l'ambassadeur leur sympathie, leur admiration et leurs regrets de le voir partir ${ }^{41}$.

38. Archives du Quai d'Orsay, série Z, Europe-Italie, 1944-I970, dépêche nº 989, Rome, I9 juillet 1967.

39. AN, 547 AP I63.

40. AN, $547 \mathrm{AP}$ I2 et $\mathrm{AP}$ I28.

4I. G. Fornari, «Le dernier des Stendhaliens", art. cité, p. 53. 
Le 17 avril 1962, Giuseppe Pella s'adresse à Gaston Palewski et lui exprime :

[...] ma joie et en même temps toute la mélancolie qui m’ont été apportés par la nouvelle de votre nomination de ministre du nouveau gouvernement français et mon regret très vif de votre départ de Rome [...]. Témoin de l'esprit de cordialité avec laquelle vous avez si sagement travaillé pour resserrer et renforcer toujours plus, chaque jour, la collaboration et la fraternité entre les deux pays. Votre mission a été couronnée du succès le plus brillant dans l'intérêt commun des deux pays ${ }^{42}$.

Si, en 1957, les choses n'ont pas été faciles pour ce nouvel ambassadeur proche du général de Gaulle, l’ambassade de Gaston Palewski à Rome constitue "un épisode heureux des relations franco-italiennes ${ }^{43}$ ». Gaston Palewski a appliqué les instructions du Quai d'Orsay, a beaucoup sillonné la Péninsule, s'est intéressé aux attraits du pays, aux villes, aux musées et à ses habitants. À son retour à Paris, il conserve un grand intérêt pour l'Italie. En 1966, il devient président de l'Association France-Italie, multiplie les réunions culturelles, organise des voyages d'études, des conférences, des tables rondes sur des questions concernant l'Italie et la France. 